\begin{tabular}{|c|c|c|}
\hline MATHRES & $\begin{array}{l}\text { Journal of Nonlinear Functional Analysis } \\
\text { Available online at http://jnfa.mathres.org }\end{array}$ & $\equiv \equiv$ \\
\hline
\end{tabular}

\title{
THE DUNKL-WIGNER TRANSFORMS ON THE REAL LINE
}

\author{
FETHI SOLTANI ${ }^{1,2}$ \\ ${ }^{1}$ Department of Mathematics, Faculty of Science, Jazan University, P.O. Box 277, Jazan 45142, Saudi Arabia \\ ${ }^{2}$ Université Tunis El Manar, Faculté des Sciences de Tunis, Laboratoire d'Analyse Mathématiques et \\ Applications, LR11ES11 2092, El Manar I, Tunis, Tunisie
}

\begin{abstract}
In this article, Calderón's reproducing formula is obtained for the Dunkl-Wigner transform $V_{g}$ and extremal functions for $V_{g}$ are also investigated.

Keywords. Dunkl-Wigner transform; Calderón's reproducing formula; Extremal function; Dunkl translation operator.
\end{abstract}

2010 Mathematics Subject Classification. 42B10, 44A20, 46F12.

\section{Introduction}

Most real-life signals of interest, such as speech, audio, seismic, radar, biomedical, and communication signals change their frequency properties over time. Therefore, a signal description by means of time-frequency analysis is often preferable to the signal's Fourier transform, which reliably yields frequency information, but without any localization in time. Time-frequency analysis has emerged as an important field in signal processing as it can be used to represen$\mathrm{t}$ time-varying signals in the time-frequency plane. Usually, the time-frequency resolution is associated with the Fourier-Wigner transform also known as the windowed Fourier transfor$\mathrm{m}$, or the short-time Fourier transform. A considerable attention has been devoted recently to discovering new mathematical formulations of the uncertainty principle for the Fourier-Wigner

E-mail address: fethisoltani10@yahoo.com.

Received December 6, 2016; Accepted March 17, 2017.

(C)2017 Mathematical Research Press 
transform, see for examples $[1,2,3,4]$ and the references therein. The most famous of them is the sharp Heisenberg-type uncertainty inequality (see [1], Theorem 5.1). An other fundamental tool in time-frequency analysis is the Dunkl-Wigner transform introduced in [5] and which is the aim of the study of this paper. Precisely, let $\mu_{k}$ be the measure on $\mathbb{R}$ given by

$$
\mathrm{d} \mu_{k}(z):=\frac{|z|^{2 k}}{2^{k+1 / 2} \Gamma(k+1 / 2)} \mathrm{d} z
$$

and $L^{p}\left(\mu_{k}\right), p \in[1, \infty]$, the space of measurable functions $f$ on $\mathbb{R}$, such that

$$
\begin{aligned}
\|f\|_{L^{p}\left(\mu_{k}\right)} & :=\left[\int_{\mathbb{R}}|f(y)|^{p} \mathrm{~d} \mu_{k}(y)\right]^{1 / p}<\infty, \quad p \in[1, \infty), \\
\|f\|_{L^{\infty}\left(\mu_{k}\right)} & :=\operatorname{ess} \sup _{y \in \mathbb{R}}|f(y)|<\infty .
\end{aligned}
$$

For $f \in L^{1}\left(\mu_{k}\right)$ the Dunkl transform of $f$ is defined (see [6]) by

$$
\mathscr{F}_{k}(f)(x):=\int_{\mathbb{R}} E_{k}(-i x y) f(y) \mathrm{d} \mu_{k}(y), \quad x \in \mathbb{R},
$$

where $E_{k}(-i x y)$ denotes the Dunkl kernel. (For more details see the next section.)

Many uncertainty principles have already been proved for the Dunkl transform $\mathscr{F}_{k}$, namely by Rösler [7] and Shimeno [8] who established the Heisenberg-type uncertainty inequality for this transform, by showing that for $f \in L^{2}\left(\mu_{k}\right)$,

$$
\|f\|_{L^{2}\left(\mu_{k}\right)}^{2} \leq \frac{2}{2 k+1}\|x f\|_{L^{2}\left(\mu_{k}\right)}\left\|y \mathscr{F}_{k}(f)\right\|_{L^{2}\left(\mu_{k}\right)} .
$$

The Dunkl translation operators $\tau_{x}, x \in \mathbb{R}$, [9] are defined on $L^{2}\left(\mu_{k}\right)$ by

$$
\mathscr{F}_{k}\left(\tau_{x} f\right)(y)=E_{k}(i x y) \mathscr{F}_{k}(f)(y), \quad y \in \mathbb{R}
$$

Let $g \in L^{2}\left(\mu_{k}\right)$. The Dunkl-Wigner transform $V_{g}$ is the mapping defined for $f \in L^{2}\left(\mu_{k}\right)$ by

$$
V_{g}(f)(x, y):=\int_{\mathbb{R}} f(t) \overline{\tau_{x} g_{k, y}(-t)} \mathrm{d} \mu_{k}(t),
$$

where

$$
g_{k, y}(z):=\mathscr{F}_{k}\left(\sqrt{\tau_{y}\left|\mathscr{F}_{k}(g)\right|^{2}}\right)(z)
$$

The contents of the paper are as follows. In Section 2, we recall some results about Dunkl transform, Dunkl translation operators and Dunkl convolution. Next, in Section 3, we study the 
Dunkl-Wigner transform $V_{g}$ and we give for it Calderón's reproducing formula in the $L^{2}\left(\mu_{k}\right)$ case. The Section 4 is devoted to study the best approximation problem

$$
\inf _{f \in H^{s}\left(\mu_{k}\right)}\left\{\eta\|f\|_{H^{s}\left(\mu_{k}\right)}^{2}+\left\|h-V_{g}(f)\right\|_{L^{2}\left(\mu_{k} \otimes \mu_{k}\right)}^{2}\right\}
$$

for an unknown function $f$, where $h \in L^{2}\left(\mu_{k} \otimes \mu_{k}\right)$ is a given function and $\eta>0, s>k+1 / 2$ are parameters with $s$ fixed throughout and $\eta$ approaching eventually 0 .

Here $H^{s}\left(\mu_{k}\right)$ is the Sobolev-Dunkl space of fractional order $s$. We provide some analysis of the minimizer $f_{\eta, h}^{*}$ of problem (1.2). Especially we use the theory of Dunkl transform, to give integral representations of $f_{\eta, h}^{*}$; and to examine the convergence rates of these type of representations. In the limit case $\eta \uparrow 0$, problem (1.2) reduces to the Tikhonov regularization problem $\inf _{f \in H^{s}\left(\mu_{k}\right)}\left\{\left\|h-V_{g}(f)\right\|_{L^{2}\left(\mu_{k} \otimes \mu_{k}\right)}^{2}\right\}$. Finally, we summarize the obtained results and describe future work in Section 5.

\section{The Dunkl convolution}

We consider the Dunkl operators $T_{k}, k \geq 0$, associated with the reflection group $G=\mathbb{Z}_{2}$ on $\mathbb{R}:$

$$
T_{k} f(x):=\frac{\mathrm{d}}{\mathrm{d} x} f(x)+k\left[\frac{f(x)-f(-x)}{x}\right] .
$$

In higher dimensions, these operators were introduced by Dunkl in [10] in connection with a generalization of the classical theory of spherical harmonics; they play a major role in various fields of mathematics $[6,11,12]$ and also in physical applications [13].

For $y \in \mathbb{R}$, the initial value problem

$$
T_{k} f(x)=y f(x), \quad f(0)=1,
$$

admits a unique analytic solution on $\mathbb{R}$, which will be denoted by $E_{k}(x y)$ and called Dunkl kernel $[11,12]$. This kernel is given explicitly by

$$
E_{k}(x y)=\mathfrak{I}_{k-1 / 2}(x y)+\frac{x y}{2 k+1} \mathfrak{I}_{k+1 / 2}(x y),
$$

where $\mathfrak{I}_{k}$ is the modified spherical Bessel function of order $k$ (see [14])

$$
\mathfrak{I}_{k}(x):=\sum_{n=0}^{\infty} \frac{\Gamma(k+1)}{n ! \Gamma(n+k+1)}\left(\frac{x}{2}\right)^{2 n}
$$


Furthermore, the Dunkl kernel $E_{k}(x y)$ has a unique analytic extension to $\mathbb{C} \times \mathbb{C}$ (see [15]), and it can be expanded in a power series in the form

$$
E_{k}(x y)=\sum_{n=0}^{\infty} \frac{(x y)^{n}}{b_{n}(k)}, \quad b_{n}(k)=\frac{2^{n}(\lfloor n / 2\rfloor) !}{\Gamma(k+1 / 2)} \Gamma(\lfloor(n+1) / 2\rfloor+k+1 / 2),
$$

where $\lfloor n / 2\rfloor$ is the integer part of $n / 2$.

The Dunkl kernel has the Laplace-type representation [16],

$$
E_{k}(x z)=\frac{\Gamma(k+1 / 2)}{\sqrt{\pi} \Gamma(k)} \int_{-1}^{1} e^{x z t}\left(1-t^{2}\right)^{k-1}(1+t) \mathrm{d} t, \quad x \in \mathbb{R}, z \in \mathbb{C} .
$$

In our case,

$$
\left|E_{k}( \pm i x y)\right| \leq 1, \quad x, y \in \mathbb{R} .
$$

The Dunkl kernel gives rise to an integral transform, which is called Dunkl transform on $\mathbb{R}$, and was introduced by Dunkl in [6], where already many basic properties were established. Dunkl's results were completed and extended later by De Jeu [12]. The Dunkl transform of a function $f$ in $L^{1}\left(\mu_{k}\right)$, is defined by

$$
\mathscr{F}_{k}(f)(x):=\int_{\mathbb{R}} E_{k}(-i x y) f(y) \mathrm{d} \mu_{k}(y), \quad x \in \mathbb{R} .
$$

We notice that $\mathscr{F}_{0}$ agrees with the Fourier transform $\mathscr{F}$ that is given by

$$
\mathscr{F}(f)(x):=(2 \pi)^{-1 / 2} \int_{\mathbb{R}} e^{-i x y} f(y) \mathrm{d} y, \quad x \in \mathbb{R} .
$$

Some of the properties of Dunkl transform $\mathscr{F}_{k}$ are collected bellow (see $\left.[6,12]\right)$.

Theorem 2.1. (i) $L^{1}-L^{\infty}$-boundedness. For all $f \in L^{1}\left(\mu_{k}\right), \mathscr{F}_{k}(f) \in L^{\infty}\left(\mu_{k}\right)$, and

$$
\left\|\mathscr{F}_{k}(f)\right\|_{L^{\infty}\left(\mu_{k}\right)} \leq\|f\|_{L^{1}\left(\mu_{k}\right)}
$$

(ii) Inversion theorem. Let $f \in L^{1}\left(\mu_{k}\right)$, such that $\mathscr{F}_{k}(f) \in L^{1}\left(\mu_{k}\right)$. Then

$$
f(x)=\mathscr{F}_{k}\left(\mathscr{F}_{k}(f)\right)(-x), \quad \text { a.e. } x \in \mathbb{R} .
$$

(iii) Plancherel theorem. The Dunkl transform $\mathscr{F}_{k}$ extends uniquely to an isometric isomorphism of $L^{2}\left(\mu_{k}\right)$ onto itself. In particular, we have

$$
\|f\|_{L^{2}\left(\mu_{k}\right)}=\left\|\mathscr{F}_{k}(f)\right\|_{L^{2}\left(\mu_{k}\right)} .
$$


(iv) Parseval theorem. For $f, g \in L^{2}\left(\mu_{k}\right)$, we have

$$
\langle f, g\rangle_{L^{2}\left(\mu_{k}\right)}=\left\langle\mathscr{F}_{k}(f), \mathscr{F}_{k}(g)\right\rangle_{L^{2}\left(\mu_{k}\right)}
$$

The Dunkl transform $\mathscr{F}_{k}$ allows us to define a generalized translation operators on $L^{2}\left(\mu_{k}\right)$ by setting

$$
\mathscr{F}_{k}\left(\tau_{x} f\right)(y)=E_{k}(i x y) \mathscr{F}_{k}(f)(y), \quad y \in \mathbb{R}
$$

It is the definition of Thangavelu and $\mathrm{Xu}$ given in [9]. It plays the role of the ordinary translation $\tau_{x} f=f(x+$.$) in \mathbb{R}$, since the Euclidean Fourier transform satisfies $\mathscr{F}\left(\tau_{x} f\right)(y)=e^{i x y} \mathscr{F}(f)(y)$. Note that from (2.1) and Theorem 2.1 (iii), the definition (2.2) makes sense, and

$$
\left\|\tau_{x} f\right\|_{L^{2}\left(\mu_{k}\right)} \leq\|f\|_{L^{2}\left(\mu_{k}\right)}, \quad f \in L^{2}\left(\mu_{k}\right)
$$

Rösler [16] introduced the Dunkl translation operators for $f$ in $L^{1}\left(\mu_{k}\right)$, by

$$
\begin{aligned}
\tau_{x} f(y) & =\int_{0}^{\pi}\left[f_{e}\left((x, y)_{\theta}\right)+f_{o}\left((x, y)_{\theta}\right) \frac{x+y}{(x, y)_{\theta}}\right] \mathrm{d} v_{x, y}(\theta), \quad(x, y) \neq(0,0) \\
\tau_{0} f(y) & =f(y),
\end{aligned}
$$

where $(x, y)_{\theta}=\left[x^{2}+y^{2}-2|x y| \cos \theta\right]^{1 / 2}$ and

$$
\mathrm{d} v_{x, y}(\theta)=\frac{\Gamma(k+1 / 2)}{\sqrt{\pi} \Gamma(k)}[1-\operatorname{sgn}(x y) \cos \theta] \sin ^{2 k-1} \theta \mathrm{d} \theta .
$$

Some of the properties of Dunkl translation operators $\tau_{x}$ are collected bellow $[9,17,18]$.

Proposition 2.2. (i) For all $p \in[1, \infty]$ and for all $x \in \mathbb{R}$, the Dunkl translation $\tau_{x}: L^{p}\left(\mu_{k}\right) \rightarrow$ $L^{p}\left(\mu_{k}\right)$ is a bounded operator, and for $f \in L^{p}\left(\mu_{k}\right)$, we have

$$
\left\|\tau_{x} f\right\|_{L^{p}\left(\mu_{k}\right)} \leq 4\|f\|_{L^{p}\left(\mu_{k}\right)}
$$

(ii) Let $f \in L^{1}\left(\mu_{k}\right)$. Then, for all $x \in \mathbb{R}$, we have

$$
\int_{\mathbb{R}} \tau_{x} f(y) \mathrm{d} \mu_{k}(y)=\int_{\mathbb{R}} f(y) \mathrm{d} \mu_{k}(y) .
$$

More details for the Dunkl translation operators are collected in $[9,17]$.

The Dunkl convolution product $*_{k}$ of two functions $f$ and $g$ in $L^{2}\left(\mu_{k}\right)$ is defined by

$$
f *_{k} g(x):=\int_{\mathbb{R}} \tau_{x} f(-y) g(y) \mathrm{d} \mu_{k}(y), \quad x \in \mathbb{R} .
$$


We notice that $*_{k}$ generalizes the convolution $*$ that is given by

$$
f * g(x):=(2 \pi)^{-1 / 2} \int_{\mathbb{R}} f(x-y) g(y) \mathrm{d} y, \quad x \in \mathbb{R} .
$$

The Proposition 2.2 allows us to establish the following properties for the Dunkl convolution on $\mathbb{R}$ (see $[9,17])$.

Proposition 2.3. (i) Assume that $p, q, r \in[1, \infty]$ such that $1 / p+1 / q=1+1 / r$. Then the map $(f, g) \rightarrow f *_{k} g$ extends to a continuous map from $L^{p}\left(\mu_{k}\right) \times L^{q}\left(\mu_{k}\right)$ to $L^{r}\left(\mu_{k}\right)$, and

$$
\left\|f *_{k} g\right\|_{L^{r}\left(\mu_{k}\right)} \leq 4\|f\|_{L^{p}\left(\mu_{k}\right)}\|g\|_{L^{q}\left(\mu_{k}\right)}
$$

(ii) For all $f \in L^{1}\left(\mu_{k}\right)$ and $g \in L^{2}\left(\mu_{k}\right)$, we have

$$
\mathscr{F}_{k}\left(f *_{k} g\right)=\mathscr{F}_{k}(f) \mathscr{F}_{k}(g)
$$

(iii) Let $f, g \in L^{2}\left(\mu_{k}\right)$. Then $f *_{k} g$ belongs to $L^{2}\left(\mu_{k}\right)$ if and only if $\mathscr{F}_{k}(f) \mathscr{F}_{k}(g)$ belongs to $L^{2}\left(\mu_{k}\right)$, and

$$
\mathscr{F}_{k}\left(f *_{k} g\right)=\mathscr{F}_{k}(f) \mathscr{F}_{k}(g), \quad \text { in the } L^{2}\left(\mu_{k}\right) \text {-case }
$$

(iv) Let $f, g \in L^{2}\left(\mu_{k}\right)$. Then

$$
\int_{\mathbb{R}}|f * g(x)|^{2} \mathrm{~d} \mu_{k}(x)=\int_{\mathbb{R}}\left|\mathscr{F}_{k}(f)(z)\right|^{2}\left|\mathscr{F}_{k}(g)(z)\right|^{2} \mathrm{~d} \mu_{k}(z),
$$

where both sides are finite or infinite.

\section{The Dunkl-Wigner transform}

Let $g \in L^{2}\left(\mu_{k}\right)$ and $y \in \mathbb{R}$. The modulation of $g$ by $y$ is the function $g_{k, y}$ defined by

$$
g_{k, y}(z):=\mathscr{F}_{k}\left(\sqrt{\tau_{y}\left|\mathscr{F}_{k}(g)\right|^{2}}\right)(z), \quad z \in \mathbb{R}
$$

Thus,

$$
\left\|g_{k, y}\right\|_{L^{2}\left(\mu_{k}\right)}=\|g\|_{L^{2}\left(\mu_{k}\right)} .
$$


Let $g \in L^{2}\left(\mu_{k}\right)$. The Fourier-Wigner transform associated to the Dunkl operators, is the mapping $V_{g}$ defined for $f \in L^{2}\left(\mu_{k}\right)$ by

$$
V_{g}(f)(x, y):=\int_{\mathbb{R}} f(t) \overline{\tau_{x} g_{k, y}(-t)} \mathrm{d} \mu_{k}(t), \quad x, y \in \mathbb{R} .
$$

As in [19], the Dunkl-Wigner transform $V_{g}$ satisfies the following properties.

Theorem 3.1. Let $f, g \in L^{2}\left(\mu_{k}\right)$. Then

(i) $V_{g}(f)(x, y)=\overline{g_{k, y}} *_{k} f(x)$.

(ii) $V_{g}(f)(x, y)=\int_{\mathbb{R}} E_{k}(i x z) \mathscr{F}_{k}(f)(z) \sqrt{\tau_{y}\left|\mathscr{F}_{k}(g)\right|^{2}(z)} \mathrm{d} \mu_{k}(z)$.

(iii) The function $V_{g}(f)$ belongs to $L^{\infty}\left(\mu_{k} \otimes \mu_{k}\right)$, and

$$
\left\|V_{g}(f)\right\|_{L^{\infty}\left(\mu_{k} \otimes \mu_{k}\right)} \leq\|f\|_{L^{2}\left(\mu_{k}\right)}\|g\|_{L^{2}\left(\mu_{k}\right)} .
$$

By using Proposition 2.2 (ii), and as in [19] we obtain the following results.

Theorem 3.2. Let $g \in L^{2}\left(\mu_{k}\right)$ be a non-zero function. Then

(i) Plancherel formula: For every $f \in L^{2}\left(\mu_{k}\right)$, we have

$$
\left\|V_{g}(f)\right\|_{L^{2}\left(\mu_{k} \otimes \mu_{k}\right)}=\|g\|_{L^{2}\left(\mu_{k}\right)}\|f\|_{L^{2}\left(\mu_{k}\right)} .
$$

(ii) Parseval formula: For every $f, h \in L^{2}\left(\mu_{k}\right)$, we have

$$
\left\langle V_{g}(f), V_{g}(h)\right\rangle_{L^{2}\left(\mu_{k} \otimes \mu_{k}\right)}=\|g\|_{L^{2}\left(\mu_{k}\right)}^{2}\langle f, h\rangle_{L^{2}\left(\mu_{k}\right)}
$$

(iii) Inversion formula: For all $f \in L^{1} \cap L^{2}\left(\mu_{k}\right)$ such that $\mathscr{F}_{k}(f) \in L^{1}\left(\mu_{k}\right)$, we have

$$
f(z)=\frac{1}{\|g\|_{L^{2}\left(\mu_{k}\right)}^{2}} \int_{\mathbb{R}} \int_{\mathbb{R}} V_{g}(f)(x, y) \overline{\tau_{z} g_{k, y}(-x)} d \mu_{k}(x) d \mu_{k}(y) .
$$

By using (1.1) and Proposition 2.2 (ii), and as in [5] we obtain the following result.

Theorem 3.3. Let $g \in L^{2}\left(\mu_{k}\right)$ be a non-zero function. Then, for $f \in L^{2}\left(\mu_{k}\right)$,

$$
\left\|x V_{g}(f)\right\|_{L^{2}\left(\mu_{k} \otimes \mu_{k}\right)}\left\|z \mathscr{F}_{k}(f)\right\|_{L^{2}\left(\mu_{k}\right)} \geq\left(k+\frac{1}{2}\right)\|f\|_{L^{2}\left(\mu_{k}\right)}^{2}\|g\|_{L^{2}\left(\mu_{k}\right)} .
$$

As in [5] we obtain the following result. 
Theorem 3.4. (Heisenberg-type uncertainty principle for $V_{g}$ ). Let $a>0$. Then there exists $a$ constant $c(k, a)>0$ such that, for all $f, g \in L^{2}\left(\mu_{k}\right)$, we have

$$
\left\||x|^{a} V_{g}(f)\right\|_{L^{2}\left(\mu_{k} \otimes \mu_{k}\right)}\left\||y|^{a} V_{g}(f)\right\|_{L^{2}\left(\mu_{k} \otimes \mu_{k}\right)} \geq c(k, a)\|f\|_{L^{2}\left(\mu_{k}\right)}^{2}\|g\|_{L^{2}\left(\mu_{k}\right)}^{2} .
$$

Theorem 3.5. Let $g \in L^{2}\left(\mu_{k}\right)$ be a non-zero function and $a, b \geq 1$. Then, for $f \in L^{2}\left(\mu_{k}\right)$,

$$
\left\||x|^{a} V_{g}(f)\right\|_{L^{2}\left(\mu_{k} \otimes \mu_{k}\right)}^{b}\left\||y|^{b} V_{g}(f)\right\|_{L^{2}\left(\mu_{k}\right)}^{a} \geq(c(k, 1))^{a b}\|f\|_{L^{2}\left(\mu_{k}\right)}^{a+b}\|g\|_{L^{2}\left(\mu_{k}\right)}^{a+b} .
$$

Proof. Let $a, b \geq 1$ and let $f \in L^{2}\left(\mu_{k}\right), f \neq 0$, such that $\left\||x|^{a} V_{g}(f)\right\|_{L^{2}\left(\mu_{k} \otimes \mu_{k}\right)}+\left\||y|^{b} V_{g}(f)\right\|_{L^{2}\left(\mu_{k}\right)}$ $<\infty$. Then for $a>1$,

$$
\left\||x|^{a} V_{g}(f)\right\|_{L^{2}\left(\mu_{k} \otimes \mu_{k}\right)}^{2 / a}\left\|V_{g}(f)\right\|_{L^{2}\left(\mu_{k} \otimes \mu_{k}\right)}^{2 / a^{\prime}}=\left\||x|^{2}\left|V_{g}(f)\right|^{\frac{2}{a}}\right\|_{L^{a}\left(\mu_{k} \otimes \mu_{k}\right)}\left\|\left|V_{g}(f)\right|^{\frac{2}{a^{\prime}}}\right\|_{L^{a^{\prime}}\left(\mu_{k} \otimes \mu_{k}\right)},
$$

where $a^{\prime}$ is defined as usual by $1 / a+1 / a^{\prime}=1$. By Hölder's inequality we get

$$
\left\||x|^{a} V_{g}(f)\right\|_{L^{2}\left(\mu_{k} \otimes \mu_{k}\right)}^{1 / a}\left\|V_{g}(f)\right\|_{L^{2}\left(\mu_{k} \otimes \mu_{k}\right)}^{1 / a^{\prime}} \geq\left\|x V_{g}(f)\right\|_{L^{2}\left(\mu_{k} \otimes \mu_{k}\right)} .
$$

Thus, for all $a \geq 1$,

$$
\left\||x|^{a} V_{g}(f)\right\|_{L^{2}\left(\mu_{k} \otimes \mu_{k}\right)}^{1 / a} \geq \frac{\left\|x V_{g}(f)\right\|_{L^{2}\left(\mu_{k} \otimes \mu_{k}\right)}}{\left\|V_{g}(f)\right\|_{L^{2}\left(\mu_{k} \otimes \mu_{k}\right)}^{1-\frac{1}{a}}},
$$

with equality if $a=1$. In the same manner, for $b \geq 1$,

$$
\left\||y|^{b} V_{g}(f)\right\|_{L^{2}\left(\mu_{k} \otimes \mu_{k}\right)}^{1 / b} \geq \frac{\left\|y V_{g}(f)\right\|_{L^{2}\left(\mu_{k} \otimes \mu_{k}\right)}}{\left\|V_{g}(f)\right\|_{L^{2}\left(\mu_{k} \otimes \mu_{k}\right)}^{1-\frac{1}{b}}}
$$

with equality if $b=1$. By (3.1) and (3.2), for all $a, b \geq 1$,

$$
\left\||x|^{a} V_{g}(f)\right\|_{L^{2}\left(\mu_{k} \otimes \mu_{k}\right)}^{1 / a}\left\||y|^{b} V_{g}(f)\right\|_{L^{2}\left(\mu_{k}\right)}^{1 / b} \geq \frac{\left\|x V_{g}(f)\right\|_{L^{2}\left(\mu_{k} \otimes \mu_{k}\right)}\left\|y V_{g}(f)\right\|_{L^{2}\left(\mu_{k}\right)}}{\left(\|f\|_{L^{2}\left(\mu_{k}\right)}\|g\|_{L^{2}\left(\mu_{k}\right)}\right)^{2-\frac{1}{a}-\frac{1}{b}}}
$$

with equality if $a=b=1$. Applying Theorem 3.4, we obtain

$$
\left\||x|^{a} V_{g}(f)\right\|_{L^{2}\left(\mu_{k} \otimes \mu_{k}\right)}^{b}\left\||y|^{b} V_{g}(f)\right\|_{L^{2}\left(\mu_{k}\right)}^{a} \geq(c(k, 1))^{a b}\|f\|_{L^{2}\left(\mu_{k}\right)}^{a+b}\|g\|_{L^{2}\left(\mu_{k}\right)}^{a+b},
$$

which completes the proof of this theorem.

The main result of this section is the following reproducing formula of Calderón's type for $V_{g}$. 
Theorem 3.6. (Calderón's reproducing formula). Let $-\infty<a<b<\infty$; and let $g \in L^{2}\left(\mu_{k}\right)$ be a non-zero function, such that $\mathscr{F}_{k}(g) \in L^{\infty}\left(\mu_{k}\right)$. Then, for $f \in L^{2}\left(\mu_{k}\right)$, the function $f_{a, b}$ given by

$$
f_{a, b}(z)=\frac{1}{\|g\|_{L^{2}\left(\mu_{k}\right)}} \int_{a}^{b} \int_{\mathbb{R}} V_{g}(f)(x, y) \overline{\tau_{z} g_{k, y}(-x)} \mathrm{d} \mu_{k}(x) \mathrm{d} \mu_{k}(y),
$$

belongs to $L^{2}\left(\mu_{k}\right)$ and satisfies

$$
\lim _{\substack{a \rightarrow-\infty \\ b \rightarrow+\infty}}\left\|f_{a, b}-f\right\|_{L^{2}\left(\mu_{k}\right)}=0
$$

Proof. From Theorem 2.1 (iii), Proposition 2.3 (iv) and Proposition 3.1 (i), we have

$$
f_{a, b}(z)=\frac{1}{\|g\|_{L^{2}\left(\mu_{k}\right)}^{2}} \int_{a}^{b} \int_{\mathbb{R}} \tau_{y}\left|\mathscr{F}_{k}(g)\right|^{2}(t) \mathscr{F}_{k}(f)(t) E_{k}(i z t) \mathrm{d} \mu_{k}(t) \mathrm{d} \mu_{k}(y) .
$$

By Fubini's theorem, we get

$$
f_{a, b}(z)=\int_{\mathbb{R}} K_{a, b}(t) \mathscr{F}_{k}(f)(t) E_{k}(i z t) \mathrm{d} \mu_{k}(t),
$$

where

$$
K_{a, b}(t)=\frac{1}{\|g\|_{L^{2}\left(\mu_{k}\right)}^{2}} \int_{a}^{b} \tau_{y}\left|\mathscr{F}_{k}(g)\right|^{2}(t) \mathrm{d} \mu_{k}(y) .
$$

It is easily to see from Proposition 2.2 (i) that $\left\|K_{a, b}\right\|_{L^{\infty}\left(\mu_{k}\right)} \leq 4$. On the other hand, by Hölder's inequality, we deduce that

$$
\left|K_{a, b}(t)\right|^{2} \leq\left.\left.\frac{\mu_{k}(a, b)}{\|g\|_{L^{2}\left(\mu_{k}\right)}^{4}} \int_{a}^{b}\left|\tau_{y}\right| \mathscr{F}_{k}(g)\right|^{2}(t)\right|^{2} \mathrm{~d} \mu_{k}(y) .
$$

Hence, by (2.3) we find

$$
\left\|K_{a, b}\right\|_{L^{2}\left(\mu_{k}\right)}^{2} \leq \frac{\left(\mu_{k}(a, b)\right)^{2}}{\|g\|_{L^{2}\left(\mu_{k}\right)}^{4}} \int_{\mathbb{R}}\left|\mathscr{F}_{k}(g)(t)\right|^{4} \mathrm{~d} \mu_{k}(t) \leq \frac{\left(\mu_{k}(a, b)\right)^{2}\left\|\mathscr{F}_{k}(g)\right\|_{L^{\infty}\left(\mu_{k}\right)}^{2}}{\|g\|_{L^{2}\left(\mu_{k}\right)}^{2}} .
$$

Thus $K_{a, b} \in L^{\infty} \cap L^{2}\left(\mu_{k}\right)$. Therefore and by (3.4) we obtain

$$
\mathscr{F}_{k}\left(f_{a, b}\right)(t)=K_{a, b}(t) \mathscr{F}_{k}(f)(t) .
$$

From this relation and Theorem 2.1 (iii), it follows that $f_{a, b} \in L^{2}\left(\mu_{k}\right)$ and

$$
\left\|f_{a, b}-f\right\|_{L^{2}\left(\mu_{k}\right)}^{2}=\int_{\mathbb{R}}\left|\mathscr{F}_{k}(f)(t)\right|^{2}\left(1-K_{a, b}(t)\right)^{2} \mathrm{~d} \mu_{k}(t) .
$$

But by Proposition 2.2 (ii) we have

$$
\lim _{\substack{a \rightarrow-\infty \\ b \rightarrow+\infty}} K_{a, b}(t)=1, \quad \text { for all } t \in \mathbb{R},
$$


and

$$
\left|\mathscr{F}_{k}(f)(t)\right|^{2}\left(1-K_{a, b}(t)\right)^{2} \leq 25\left|\mathscr{F}_{k}(f)(t)\right|^{2}, \quad \text { for all } t \in \mathbb{R} \text {. }
$$

So, the relation (3.3) follows from the dominated convergence theorem.

\section{Extremal functions for the mapping $V_{g}$}

In this section, by using the theory of Dunkl transform [12], and building on the ideas of Saitoh [20, 21, 22], we examine the extremal function of the problem (1.2).

Let $s \geq 0$. We define the Sobolev-Dunkl space of order $s$, that will be denoted $H^{s}\left(\mu_{k}\right)$, as the set of all $f \in L^{2}\left(\mu_{k}\right)$ such that $\left(1+z^{2}\right)^{s / 2} \mathscr{F}_{k}(f) \in L^{2}\left(\mu_{k}\right)$. The space $H^{s}\left(\mu_{k}\right)$ provided with the inner product

$$
\langle f, g\rangle_{H^{s}\left(\mu_{k}\right)}=\int_{\mathbb{R}}\left(1+z^{2}\right)^{s} \mathscr{F}_{k}(f)(z) \overline{\mathscr{F}_{k}(g)(z)} \mathrm{d} \mu_{k}(z)
$$

and the norm

$$
\|f\|_{H^{s}\left(\mu_{k}\right)}=\left[\int_{\mathbb{R}}\left(1+z^{2}\right)^{s}\left|\mathscr{F}_{k}(f)(z)\right|^{2} \mathrm{~d} \mu_{k}(z)\right]^{1 / 2} .
$$

Let $\eta>0$. We denote by $\langle., .\rangle_{\eta, H^{s}\left(\mu_{k}\right)}$ the inner product defined on the space $H^{s}\left(\mu_{k}\right)$ by

$$
\langle f, h\rangle_{\eta, H^{s}\left(\mu_{k}\right)}:=\eta\langle f, h\rangle_{H^{s}\left(\mu_{k}\right)}+\left\langle V_{g}(f), V_{g}(h)\right\rangle_{L^{2}\left(\mu_{k} \otimes \mu_{k}\right)},
$$

and the norm $\|f\|_{\eta, H^{s}\left(\mu_{k}\right)}:=\sqrt{\langle f, f\rangle_{\eta, H^{s}\left(\mu_{k}\right)}}$.

Next, we suppose that $g \in L^{2}\left(\mu_{k}\right)$. By Theorem 3.2 (ii), the inner product $\langle., .\rangle_{\eta, H^{s}\left(\mu_{k}\right)}$ can be written

$$
\langle f, h\rangle_{\eta, H^{s}\left(\mu_{k}\right)}=\eta\langle f, h\rangle_{H^{s}\left(\mu_{k}\right)}+\|g\|_{L^{2}\left(\mu_{k}\right)}^{2}\langle f, h\rangle_{L^{2}\left(\mu_{k}\right)} .
$$

Let $\eta>0$ and $s>k+1 / 2$ and let $g \in L^{2}\left(\mu_{k}\right)$. The space $H^{s}\left(\mu_{k}\right)$, equipped with the norm $\|\cdot\|_{\eta, H^{s}\left(\mu_{k}\right)}$ has the reproducing kernel

$$
K_{\eta, g}(x, y)=\int_{\mathbb{R}} \frac{E_{k}(i x z) E_{k}(-i y z)}{\eta\left(1+z^{2}\right)^{s}+\|g\|_{L^{2}\left(\mu_{k}\right)}^{2}} \mathrm{~d} \mu_{k}(z),
$$

that is,

(i) For all $y \in \mathbb{R}$, the function $x \rightarrow K_{\eta, g}(x, y)$ belongs to $H^{s}\left(\mu_{k}\right)$.

(ii) The reproducing property: for all $f \in H^{s}\left(\mu_{k}\right)$ and $y \in \mathbb{R}$,

$$
\left\langle f, K_{\eta, g}(., y)\right\rangle_{\eta, H^{s}\left(\mu_{k}\right)}=f(y) .
$$


The main result of this section can then be stated as follows.

Theorem 4.1. Let $s>k+1 / 2$ and $g \in L^{2}\left(\mu_{k}\right)$. For any $h \in L^{2}\left(\mu_{k} \otimes \mu_{k}\right)$ and for any $\eta>0$, the problem

$$
\inf _{f \in H^{s}\left(\mu_{k}\right)}\left\{\eta\|f\|_{H^{s}\left(\mu_{k}\right)}^{2}+\left\|h-V_{g}(f)\right\|_{L^{2}\left(\mu_{k} \otimes \mu_{k}\right)}^{2}\right\}
$$

has a unique extremal function $f_{\eta, h}^{*}$ given by

$$
f_{\eta, h}^{*}(y)=\int_{\mathbb{R}} \int_{\mathbb{R}} h(x, t) Q_{s}(x, y, t) \mathrm{d} \mu_{k}(t) \mathrm{d} \mu_{k}(x),
$$

where

$$
Q_{s}(x, y, t)=\int_{\mathbb{R}} \frac{E_{k}(-i x z) E_{k}(i y z) \sqrt{\tau_{t}\left|\mathscr{F}_{k}(g)\right|^{2}(z)}}{\eta\left(1+z^{2}\right)^{s}+\|g\|_{L^{2}\left(\mu_{k}\right)}^{2}} \mathrm{~d} \mu_{k}(z) .
$$

Proof. Problem (4.1) is solved elementarily by finding the roots of the first derivative $D \Phi$ of the quadratic and strictly convex function

$$
\Phi(f)=\eta\|f\|_{H^{s}\left(\mu_{k}\right)}^{2}+\left\|h-V_{g}(f)\right\|_{L^{2}\left(\mu_{k} \otimes \mu_{k}\right)}^{2} .
$$

Note that for convex functions the equation $D \Phi(f)=0$ is a necessary and sufficient condition for the minimum at $f$. The calculation provides

$$
D \Phi(f)=2 \eta f+2 V_{g}^{*}\left(V_{g}(f)-h\right)
$$

where $V_{g}^{*}: L^{2}\left(\mu_{k} \otimes \mu_{k}\right) \rightarrow H^{s}\left(\mu_{k}\right)$ is the adjoint of $V_{g}$. Then

$$
f_{\eta, h}^{*}(y)=\left(\eta I+V_{g}^{*} V_{g}\right)^{-1} V_{g}^{*}(h)(y) .
$$

On the other hand, we deduce that

$$
\begin{aligned}
f_{\eta, h}^{*}(y) & =\left\langle\left(\eta I+V_{g}^{*} V_{g}\right)^{-1} V_{g}^{*}(h), K_{1,0}(., y)\right\rangle_{H_{s}\left(\mu_{k}\right)} \\
& =\left\langle V_{g}^{*}(h),\left(\eta I+V_{g}^{*} V_{g}\right)^{-1} K_{1,0}(., y)\right\rangle_{H_{s}\left(\mu_{k}\right)} \\
& =\left\langle V_{g}^{*}(h), K_{\eta, g}(., y)\right\rangle_{H_{s}\left(\mu_{k}\right)} .
\end{aligned}
$$

Hence, one has

$$
f_{\eta, h}^{*}(y)=\left\langle h, V_{g}\left(K_{\eta, g}(., y)\right)\right\rangle_{L^{2}\left(\mu_{k} \otimes \mu_{k}\right)} .
$$

From (2.1), the function $\Phi_{y}: z \rightarrow \frac{E_{k}(-i y z)}{\eta\left(1+z^{2}\right)^{s}+\|g\|_{L^{2}\left(\mu_{k}\right)}^{2}}$ belongs to $L^{1} \cap L^{2}\left(\mu_{k}\right)$. Then from Theorem 2.1 (iii), it follows that $K_{\eta, g}(., y)$ belongs to $L^{2}\left(\mu_{k}\right)$, and

$$
\mathscr{F}_{k}\left(K_{\eta, g}(., y)\right)(z)=\frac{E_{k}(-i y z)}{\eta\left(1+z^{2}\right)^{s}+\|g\|_{L^{2}\left(\mu_{k}\right)}^{2}}, \quad z \in \mathbb{R} .
$$


By Proposition 3.1 (ii) and (4.3), we have

$$
\begin{aligned}
V_{g}\left(K_{\eta, g}(., y)\right)(x, t) & =\int_{\mathbb{R}} E_{k}(i x, z) \mathscr{F}_{k}\left(K_{\eta, g}(., y)\right)(z) \sqrt{\tau_{t}\left|\mathscr{F}_{k}(g)\right|^{2}(z)} \mathrm{d} \mu_{k}(z) \\
& =\int_{\mathbb{R}} \frac{E_{k}(i x z) E_{k}(-i y z) \sqrt{\tau_{t}\left|\mathscr{F}_{k}(g)\right|^{2}(z)}}{\eta\left(1+z^{2}\right)^{s}+\|g\|_{L^{2}\left(\mu_{k}\right)}^{2}} \mathrm{~d} \mu_{k}(z) .
\end{aligned}
$$

This clearly yields the result.

As in the same of [19] for the radial case, the extremal function $f_{\eta, h}^{*}$ satisfies the following properties.

Theorem 4.2. Let $s>k+1 / 2$ and $g \in L^{2}\left(\mu_{k}\right)$. For any $h \in L^{2}\left(\mu_{k} \otimes \mu_{k}\right)$ and for any $\eta>0$, we have

(i) $f_{\eta, h}^{*}(y)=\int_{\mathbb{R}} \int_{\mathbb{R}} \frac{E_{k}(i y z) \sqrt{\tau_{t}\left|\mathscr{F}_{k}(g)\right|^{2}(z)} \mathscr{F}_{k}(h(., t))(z)}{\eta\left(1+z^{2}\right)^{s}+\|g\|_{L^{2}\left(\mu_{k}\right)}^{2}} \mathrm{~d} \mu_{k}(t) \mathrm{d} \mu_{k}(z)$.

(ii) $\mathscr{F}_{k}\left(f_{\eta, h}^{*}\right)(z)=\frac{\int_{\mathbb{R}} \sqrt{\tau_{t}\left|\mathscr{F}_{k}(g)\right|^{2}(z)} \mathscr{F}_{k}(h(., t))(z) \mathrm{d} \mu_{k}(t)}{\eta\left(1+z^{2}\right)^{s}+\|g\|_{L^{2}\left(\mu_{k}\right)}^{2}}$.

Theorem 4.3. Let $s>k+1 / 2$ and $g \in L^{2}\left(\mu_{k}\right)$. For $h=V_{g}(f), f \in H^{s}\left(\mu_{k}\right)$, we have

(i) $\lim _{\eta \rightarrow 0^{+}}\left\|f_{\eta, V_{g}(f)}^{*}-f\right\|_{L^{\infty}\left(\mu_{k}\right)}=0$.

(ii) $\lim _{\eta \rightarrow 0^{+}}\left\|f_{\eta, V_{g}(f)}^{*}-f\right\|_{H^{s}\left(\mu_{k}\right)}=0$.

Proof. (i) From Proposition 3.1 (ii), we have

$$
\mathscr{F}_{k}\left(V_{g}(f)(., t)(z)=\sqrt{\tau_{t}\left|\mathscr{F}_{k}(g)\right|^{2}(z)} \mathscr{F}_{k}(f)(z)\right.
$$

Hence, by Proposition 2.2 (ii) and Theorem 4.2 (i), we obtain

$$
f_{\eta, V_{g}(f)}^{*}(y)=\|g\|_{L^{2}\left(\mu_{k}\right)}^{2} \int_{\mathbb{R}} \frac{E_{k}(i y z) \mathscr{F}_{k}(f)(z)}{\eta\left(1+z^{2}\right)^{s}+\|g\|_{L^{2}\left(\mu_{k}\right)}^{2}} \mathrm{~d} \mu_{k}(z) .
$$

Since $\mathscr{F}_{k}(f) \in L^{1} \cap L^{2}\left(\mu_{k}\right)$, then by (4.4) and Theorem 2.1 (ii), we deduce that

$$
f_{\eta, V_{g}(f)}^{*}(y)-f(y)=\int_{\mathbb{R}} \frac{-\eta\left(1+z^{2}\right)^{s} \mathscr{F}_{k}(f)(z)}{\eta\left(1+z^{2}\right)^{s}+\|g\|_{L^{2}\left(\mu_{k}\right)}^{2}} E_{k}(i y z) \mathrm{d} \mu_{k}(z) .
$$

So, one has

$$
\left\|f_{\eta, V_{g}(f)}^{*}-f\right\|_{L^{\infty}\left(\mu_{k}\right)} \leq \int_{\mathbb{R}^{d}} \frac{\eta\left(1+z^{2}\right)^{s}\left|\mathscr{F}_{k}(f)(z)\right|}{\eta\left(1+z^{2}\right)^{s}+\|g\|_{L^{2}\left(\mu_{k}\right)}^{2}} \mathrm{~d} \mu_{k}(z)
$$


Again, by dominated convergence theorem and the fact that

$$
\frac{\eta\left(1+z^{2}\right)^{s}\left|\mathscr{F}_{k}(f)(z)\right|}{\eta\left(1+z^{2}\right)^{s}+\|g\|_{L^{2}\left(\mu_{k}\right)}^{2}} \leq\left|\mathscr{F}_{k}(f)(z)\right|
$$

we deduce (i).

(ii) As in (i), from Theorem 4.2 (ii), we have

$$
\mathscr{F}_{k}\left(f_{\eta, V_{g}(f)}^{*}\right)(z)=\frac{\|g\|_{L^{2}\left(\mu_{k}\right)}^{2} \mathscr{F}_{k}(f)(z)}{\eta\left(1+z^{2}\right)^{s}+\|g\|_{L^{2}\left(\mu_{k}\right)}^{2}},
$$

and

$$
\mathscr{F}_{k}\left(f_{\eta, V_{g}(f)}^{*}-f\right)(z)=\frac{-\eta\left(1+z^{2}\right)^{s} \mathscr{F}_{k}(f)(z)}{\eta\left(1+z^{2}\right)^{s}+\|g\|_{L^{2}\left(\mu_{k}\right)}^{2}} .
$$

Consequently, we have

$$
\left\|f_{\eta, V_{g}(f)}^{*}-f\right\|_{H^{s}\left(\mu_{k}\right)}^{2}=\int_{\mathbb{R}^{d}} \frac{\eta^{2}\left(1+z^{2}\right)^{3 s}\left|\mathscr{F}_{k}(f)(z)\right|^{2}}{\left[\eta\left(1+z^{2}\right)^{s}+\|g\|_{L^{2}\left(\mu_{k}\right)}^{2}\right]^{2}} \mathrm{~d} \mu_{k}(z) .
$$

Using the dominated convergence theorem and the fact that

$$
\frac{\eta^{2}\left(1+z^{2}\right)^{3 s}\left|\mathscr{F}_{k}(f)(z)\right|^{2}}{\left[\eta\left(1+z^{2}\right)^{s}+\|g\|_{L^{2}\left(\mu_{k}\right)}^{2}\right]^{2}} \leq\left(1+z^{2}\right)^{s}\left|\mathscr{F}_{k}(f)(z)\right|^{2}
$$

we deduce (ii).

\section{Conclusions and perspectives}

We investigated the Dunkl-Wiener transform on $\mathbb{R} \times \mathbb{R}$, which generalizes the windowed Fourier transform and the windowed Hankel transform. Some results related to the DunklWiener transform on $\mathbb{R} \times \mathbb{R}$ are proven, such as, Calderón's reproducing formula, Heisenbergtype uncertainty principle and a best approximation problem. With less restricted techniques, these results are obtained for the windowed Fourier transform, the windowed Hankel transform and the Dunkl-Wiener transform on $\mathbb{R}^{d} \times \mathbb{R}^{d}$ (for the radial case). However, the general case concerning the Dunkl-Wiener transform on $\mathbb{R}^{d} \times \mathbb{R}^{d}$ will be an open topic (difficult). This topic requires more details for the Dunkl translation operators in $d$-dimensions. 


\section{REFERENCES}

[1] A. Bonami, B. Demange, P. Jaming, Hermite functions and uncertainty principles for the Fourier and the windowed Fourier transforms, Rev. Mat. Iberoamericana 19 (2003), 23-55.

[2] B. Demange, Uncertainty principles for the ambiguity function, J. Lond. Math. Soc. 72 (2005), 717-730.

[3] K. Gröchenig, Uncertainty principles for time-frequency representations. In: Feichtinger HG, Strohmer T, editors. Advances in Gabor analysis. Boston, MA: Birkhuser Boston; 11-30, 2003.

[4] E. Wilczok, New uncertainty principles for the continuous Gabor transform and the continuous wavelet transform, Doc. Math. 5 (2000) 201-226.

[5] F. Soltani, Uncertainty principles for the Dunkl-Wigner transforms, J. Oper. 2016 (2016), Article ID 7637346.

[6] C.F. Dunkl, Hankel transforms associated to finite reflection groups, Contemporary Math. 138 (1992), 123138.

[7] M. Rösler, An uncertainty principle for the Dunkl transform, Bull. Austral. Math. Soc. 59 (1999), 353-360.

[8] N. Shimeno, A note on the uncertainty principle for the Dunkl transform, J. Math. Sci. Univ. Tokyo 8 (2001), $33-42$.

[9] S. Thangavelu, Y. Xu, Convolution operator and maximal function for the Dunkl transform, J. Anal. Math. 97 (2005), 25-55.

[10] C.F. Dunkl, Differential-difference operators associated to reflection groups, Trans. Amer. Math. Soc. 311 (1989), 167-183.

[11] C.F. Dunkl, Integral kernels with reflection group invariance, Canadian J. Math. 43 (1991), 1213-1227.

[12] M.F.E.de Jeu, The Dunkl transform, Invent. Math. 113 (1993), 147-162.

[13] L. Lapointe, L. Vinet, Exact operator solution of the Calogero- Sutherland model, Commun. Math. Phys. 178 (1996), 425-452.

[14] G.N. Watson, A treatise on theory of Bessel functions, Cambridge University Press, Cambridge, 1966.

[15] E.M. Opdam, Dunkl operators, Bessel functions and the discriminant of a finite Coxeter group, Compositio Math. 85 (1993), 333-373.

[16] M. Rösler, Bessel-type signed hypergroups on $\mathbb{R}$, in Probability Measures on Groups and Related Structures, XI (Oberwolfach, 1994), Editors H. Heyer and A. Mukherjea, Oberwolfach, 1994, World Sci. Publ., River Edge, NJ, 292-304, 1995.

[17] F. Soltani, $L^{p}$-Fourier multipliers for the Dunkl operator on the real line, J. Funct. Anal. 209 (2004), 16-35.

[18] S. Thangavelu, Y. Xu, Riesz transform and Riesz potentials for Dunkl transform, J. Comput. Appl. Math. 199 (2007), 181-195.

[19] F. Soltani, Reproducing inversion formulas for the Dunkl-Wigner transforms, CUBO Math. J. 17 (2015), $1-14$.

[20] S. Saitoh, The Weierstrass transform and an isometry in the heat equation, Appl. Anal. 16 (1983), 1-6.

[21] S. Saitoh, Approximate real inversion formulas of the Gaussian convolution, Appl. Anal. 83 (2004), 727-733. 
[22] S. Saitoh, Best approximation, Tikhonov regularization and reproducing kernels, Kodai Math. J. 28 (2005), 359-367. 